\title{
Provision of cancer care during the COVID-19 pandemic
}

James Spicer ${ }^{1,2 凶}$, Charlotte Chamberlain ${ }^{3}$ and Sophie Papa (i) ${ }^{1,2}$

Health-care services are rapidly transforming their organization and workforce in response to the coronavirus disease 2019 (COVID-19) pandemic. These changes, and a desire to mitigate infection risk, are having profound effects on other vital aspects of care, including the care of patients with cancer. Difficult decisions are being made regarding the prioritization of both active treatments and palliative care, despite limited evidence that cancer is an independent risk factor for infection and mortality.

\section{COVID-19 outcomes in patients with cancer}

According to published data from China reported by Liang et al. ${ }^{1}$, cancer seems to have comparable associations with an increased risk of death from coronavirus disease 2019 (COVID-19) to those of other comorbidities, such as chronic respiratory disease and hypertension. However, whether the multivariate analysis that suggested this association accounted for the potential confounding effects of noncancer comorbidities remains unclear. Conclusions thus far have had to be drawn from the analysis of data from small subgroups of patients with cancer in much larger population-based series. In a correspondence ${ }^{2}$, the authors highlight that half of the patients with cancer included in the analysis by Liang et al. ${ }^{1}$ were diagnosed $>4$ years before SARS-CoV-2 infection, suggesting that these patients might not have active cancer. The observations by Liang et al. ${ }^{1}$ might, therefore, not be generalizable to patients with cancer requiring ongoing treatment and/or management who develop COVID-19. In comparison to those without cancer, as expected, the patients with cancer included in the study by Liang et al. ${ }^{1}$ were older (mean age of 63 years versus 49 years), and this might be a powerful confounder given the strong correlation between advanced age and death from COVID-19 (REF. $\left.{ }^{3}\right)$. In other reports of risk factors in patients with COVID-19 (REFS ${ }^{4,5}$ ), the authors have not analysed cancer as a comorbidity, presumably owing to small sample sizes and the generally low prevalence of cancer at the population level.

In marked contrast to data from China, a preliminary report from Italy indicates that $20 \%$ of patients with COVID-19 had a diagnosis of cancer in the preceding 5 years $^{6}$. However, in this analysis, the identification of comorbidities was confined to patients who died of COVID-19; therefore, distinguishing between cancer as an independent risk factor for developing COVID-19 or for poor outcomes is not possible. Analysis of incidence and outcomes specifically in patients with cancer and
COVID-19 will enable a clearer understanding of the associated risks.

\section{Acute management and palliative care}

The presenting features of COVID-19, such as fever, fatigue, dyspnoea and arthralgia/myalgia, are often similar to those of patients with cancer, especially those receiving treatment. Therefore, the recognition of COVID-19 symptoms in such patients can be problematic. Guidelines provided by the UK National Institute for Health and Care Excellence (NICE) state that if the differential diagnosis includes the possibility of neutropenic sepsis, this must first be excluded as this is the most immediately life-threatening condition ${ }^{7}$. In the inpatient setting, those without suspected COVID-19 must be segregated from those with established or suspected COVID-19. Furthermore, a high proportion of hospitalized patients with COVID-19 will require respiratory support. For patients with cancer, the appropriate level of escalation of care for COVID-19 symptoms is likely to be affected by the patient's expected survival duration based on disease stage, treatment history and pre-morbid performance status. Carers need to encourage their patients to discuss in advance their priorities for treatment escalation because critical care, including ventilatory support, is likely to be explicitly rationed in most health-care systems and is unlikely to be successful in many patients with advanced-stage cancer.

Reduced availability of community symptom support might result in an increase in hospital admissions at a time when many health-care systems are least able to manage such increases. Already under-resourced palliative care teams are likely to face staffing crises just when demand increases exponentially. These issues have implications for patients with cancer and their families affected by COVID-19, as well as for those with life-limiting cancer without COVID-19, whose symptoms might deteriorate as a result of reduced health-care provision. 
COVID-19 also presents a barrier to hospice admission and, therefore, oncologists are likely to be required to manage certain complex symptoms that would otherwise benefit from the involvement of palliative care teams. Guidance on the adaptation of palliative care to the demands of the COVID-19 pandemic has been provided by the Center to Advance Palliative Care, and the European Association for Palliative Care have provided advice for non-specialists on counselling patients who have become critically unwell owing to COVID-19.

Practical end-of-life care considerations in the context of the COVID-19 pandemic include limitations of visitor access to patients, even for close relatives, owing to concerns regarding viral transmission. These considerations might be compounded by restricted supplies of personal protective equipment in certain health-care systems and restricted mortuary and funeral arrangements enforced by capacity constraints and/or public health measures. Thus, the negative psychological sequelae of the pandemic, which could lead to suboptimal care, symptom management and end-of-life care, might be substantial for patients with cancer, their families and/or their carers.

\section{Service reconfiguration during the pandemic}

Social distancing, lockdowns and curfews are creating major long-term issues for both curative and palliative oncology care. Patients need to be managed, as far as possible, without visits to hospitals or other treatment centres in order to minimize infection risk and by applying the precautionary principle as more data on infection risks are gathered ${ }^{8}$. This aspect, in addition to the re-deployment of resources to acute COVID-19 care, creates problems with the delivery of timely treatment, the continuity of care and access to clinical trials, most of which are now closed to recruitment. Patients with fever or respiratory symptoms need to be triaged on arrival at the cancer centre in order to reduce the risk of exposure to other patients and staff. Access to SARS-CoV-2 testing for patients can help with this triage. Testing of staff, a problem even in the health-care systems of many economically developed countries, defines a return-to-work pathway that is essential for maintenance of a stable workforce.

Remote review by telephone or video-link can be used in routine outpatient practice, when the benefits of attending hospital in person are judged to be outweighed by the risks of exposure to infection ${ }^{7}$. Other methods of reducing the need for patients to travel include home deliveries of oral medicines, local and/or remote availability of routine clinical testing, and remote consenting and rationalization of investigations. Increased operating hours of outpatient services and the provision of cancer-specific acute evaluation capabilities can all help in relieving the pressures placed on emergency departments. Blood product shortages arising from societal effects on donations are likely and can be mitigated in part by raising the thresholds for transfusion and through greater use of erythropoietin. Oncology inpatient activity is also likely to be fundamentally and rapidly affected, both owing to the necessity of reallocating dedicated beds for general respiratory support and as a result of a proportion of the outpatient population requiring admission with the co-diagnoses of cancer and COVID-19.

Effects on infrastructure and staffing levels in cancer services affected by COVID-19 have led to some of the most difficult decisions. As highlighted in the UK National Health Service (NHS) guidelines in this area, constraints on access to operating theatres and anaesthetic support might be a particular problem for patients requiring surgery or brachytherapy ${ }^{9}$. Prioritization of treatment for patients with cancer should, therefore, be formalized to ensure that those with the most important implications for oncological outcomes are preferentially delivered when resources become constrained ${ }^{7,9}$. Prioritization of treatment implies rationing of care, and these decisions should, where possible, be taken as a team and be discussed fully with the patient. When treatment plans deviate from standard practice, this should be carefully documented. Prioritization must also take into account and balance the relative benefits of treatment against the estimated increased risks of infection. Although we recognize the substantial risks of viral infection to all members of the population, COVID-19 will not harm every patient with cancer.

Treatment delays, the introduction of regimens requiring less-frequent administration and hypofractionated radiotherapy are all important components of a pandemic mitigation strategy for patients who nonetheless require treatment for other conditions. Delays in the delivery of palliative interventions, which are at risk of low-level prioritization in this context, might lead to worsening symptoms, a loss of any possible treatment opportunities and hospital admission for symptom control at a time when inpatient resources are least available. For most patients with solid tumours, the survival benefits obtained with adjuvant treatments must be offset against the risks posed by regular clinic attendance for myelosuppressive therapy: addition of growth factor support in this setting is now widely advocated ${ }^{7}$. Prioritization of surgical interventions is also important, as is the consideration of radical alternatives to surgery, especially radiotherapy, and safe methods of delaying the need for surgery such as in women with certain types of breast cancer using endocrine therapy, or in men with prostate cancer using androgen-deprivation therapy. Further data are required in order to understand how best to extrapolate the current limited understanding of the immunology of SARS-CoV-2 to the effects of anticancer immunotherapy. A systematic review revealed little evidence that immuno-modulatory agents, such as steroids, cytotoxic agents, tacrolimus, JAK inhibitors and biologics, including anti-TNF antibodies, increase the risk of severe COVID-19 symptoms ${ }^{10}$. The use of anti-PD-1 antibodies might, theoretically, limit the extent of $\mathrm{T}$ cell exhaustion and thus promote viral elimination, or could potentially contribute to the excessive inflammatory response to viral infection.

\section{Conclusions}

Data emerging from countries affected early in the COVID-19 pandemic have, thus far, provided little evidence to improve our understanding of the effects of 
having cancer on the risk of either SARS-CoV-2 infection or on the outcomes of COVID-19. Prospective collection of data on the outcomes of patients with cancer and COVID-19 across all age ranges, tumour types and treatment modalities will enable more robust conclusions to be drawn. These data need to be rapidly collated and shared. Reassessment of the relative risks and benefits of cancer therapies in the context of the COVID-19 pandemic is fundamental to the continued safe delivery of these treatments. At the same time, the redeployment of infrastructure and personnel across a health-care system might in itself also contribute to inferior cancer outcomes. Every effort should be made to avoid compromising the delivery of evidence-based cancer care when infection risks are outweighed by the benefits of treatment.

1. Liang, W. et al. Cancer patients in SARS-CoV-2 infection: a nationwide analysis in China. Lancet Oncol. 21, 335-337 (2020).

2. Xia, Y., Jin, R., Zhao, J., Li, W. \& Shen, H. Risk of COVID-19 for cancer patients. Lancet Oncol. 21, e180 (2020).

3. Wu, Z. \& Mcgoogan, J. M. Characteristics of and important lessons from the coronavirus disease 2019 (COVID-19) outbreak in china. JAMA 323, 1239-1242 (2020).

4. Grasselli, G. et al. Baseline characteristics and outcomes of 1591 patients infected with SARS-CoV-2 admitted to ICUs of the Lombardy region, Italy. JAMA https://doi.org/10.1001/ jama.2020.5394 (2020).
5. Zhou, F. et al. Clinical course and risk factors for mortality of adult inpatients with COVID-19 in Wuhan, China: a retrospective cohort study. Lancet 395, 1054-1062 (2020).

6. Palmieri et al. Characteristics of COVID-19 patients dying in Italy. Report based on available data on March 20th, 2020. https://www. epicentro.iss.it/coronavirus/bollettino/Report-COVID-2019_20_ marzo_eng.pdf (2020).

7. The UK National Institute of Health and Care Excellence. COVID-19 rapid guideline: delivery of systemic anticancer treatments. https:// www.nice.org.uk/guidance/ng 161 (2020).

8. Hanna, T. P., Evans, G. A. \& Booth, C. M. Cancer, COVID-19 and the precautionary principle: prioritizing treatment during a global pandemic. Nat. Rev. Clin. Oncol. https://doi.org/10.1038/ s41571-020-0362-6 (2020).

9. NHS England. Clinical guide for the management of non-coronavirus patients requiring acute treatment. https://www.england.nhs.uk/ coronavirus/wp-content/uploads/sites/52/2020/03/specialty-guideacute-treatment-cancer-23-march-2020.pdf (2020).

10. Russell, B. et al. Associations between immune-suppressive and stimulating drugs and novel Covid-19 - a systematic review of current evidence. eCancer 14, 1022 (2020).

\section{Acknowledgements}

The authors thank colleagues for discussions and assistance in preparing this commentary, especially Richard Sullivan and Dan Hughes at King's College London, and fellow clinicians at Guy's \& St Thomas' NHS Foundation Trust.

Competing interests

The authors declare no competing interests.

\section{RELATED LINKS}

Center to Advance Palliative Care: https://www.capc.org/toolkits/ covid-19-response-resources

European Association for Palliative Care: https://www.eapcnet.eu/ publications/coronavirus-and-the-palliative-care-response 